of meat-poisoning, certain gas producing so-called "typhoid" bacilli, the B. psittacosis, and even the B. icteroides of Sanarelli, are all to be regarded as intermediates between the colon and the typhosus group; while in the same series there are other bacilli which, so far as their characteristics place them in the series, should stand beyond the B. typhosus, thus out-typhoiding the typhoid bacillus itself. The clinical interest of all this, however, centres itself on the fact that we may meet with cases which resemble, or indeed appear identical with typhoid, but yet appear to be caused by an intermediate bacillus, thus perhaps explaining the occasional discrepancies reported in regard to the Widal reaction. In fact, "practically all the cases of paratyphoid infection passed clinically for typhoid fever without a Widal reaction, until careful bacteriological examination revealed their true nature." The authors then go minutely into the various bacteriological differences between these organs, into all of which we need not now enter, sufficient to point out that certain anomalous cases of what appear to be typhoid fever are possibly cases of infection by organisms intermediate between that: organism and the B. coli.

1 American Jour nal of Medical Sciences, June, 1902.

\section{GOLD MINERS' PHTHISIS.}

Among the newer forms of industrial diseases we must place gold miners' phthisis as met with in the deep gold mines in the Transvaal. Owing to the closing of the mines and the continuance of the war, large numbers of miners who had gone out from the colliery districts of Northumberland have returned to their old homes, and Dr. Thomas Oliver ${ }^{1}$ says that many of these men have presented on their arrival the appearance of men broken down in health and have sooner or later succumbed to pulmonary disease. He has been able moreover to show that their lung affection has assumed the characters of a veritable pulmonary fibrosis. Gold miners' disease of the lungs is due, he says, to the inhalation of dust, and is a malady akin to stonemasons' and steel-grinders' phthisis. Like all the pneumoconioses, it is slowly developed. The earliest symptom is cough, especially in the morning, then comes shortwindedness. So long as the patient keeps quiet the breathing may be perfectly easy, but the slightest effort, or walking in the face of a wind, causes the breathing to become difficult and noisy as in asthma. By degrees the appe'tite is lost and emaciation becomes slowly progressive, but there is none of the night-sweating which is so characteristic of ordinary tuberculous phthisis. From first to last it is an afebrile and a painless affection. It seems to commence as a peri-bronchitis, and this is followed by an increase of the fibro-connective tissue of the lung, although, as is the case in stonemasons' phthisis, it may have a tuberculous lesion engrafted upon it. Two risks to health are incurred by the men engaged in the gold mines, besides the possible dangers which are run in the abstraction of the gold from the rock by means of what is known as the "cyanide process." One danger arises from breathing the poisonous gases which result from the large quantities of high explosives used in sinking the shafts, and from the other impurities which accumulate in the air at the bottom of these deep shafts owing to the difficulty of ventilating them.
But it is in the act of mining that the danger of pulmonary disease arises. Gold mining is a very dusty process, the rock is hard and has to be drilled by machinery, and it is the dry fine dust given off in drilling the rock that is the cause of lung trouble in gold miners. A man may work five or six years before he is rendered hors de combat by the dust. Gold miners' phthisis therefore kills men when they are still comparatively young, usually before they reach the age of forty years.

1 Lancet, June 14.

\section{PREGNANCY AFTER DOUBLE OVARIOTOMY.}

Ar the last meeting of the Obstetrical Society, a case was reported by $\mathrm{Mr}$. Alban Doran in which pregnancy had occurred after removal of both ovaries for cystic tumour. Mr. Doran said that, although it was not difficult to extirpate all ovarian tissue when the tumour was pedunculated, the case was different when the base of the cyst burrowed and lay close to the uterus. The same difficulty arose also with inflamed adherent appendages, and very often also with an ovary removed to check the growth of a uterine fibroid. The operator dared not cut the pedicle sufficiently short to remove all ovarian tissue, for he knew that if he did so the ligature would probably slip. Hence it was not very hard to understand why in some cases menstruation or even pregnancy occurred after removal of both ovaries. In his own case the ovarian ligament, with some healthy tissue, had probably been flattened against the uterus, and had thus escaped the ligature. This case, however, and others mentioned in the course of the discussion, raised the very interesting question how far the application of a ligature to both Fallopian tubes could be trusted to ensure sterility. Several cases were mentioned which went to show that the mere application of a silk ligature to the Fallopian tubes could not be depended upon to permanently obliterate their lumina, and thus prove an obstacle to pregnancy. Mr. BlandSutton said that when it was desirable to sterilise a patient, he always tied the tubes in two places and exsected a piece of the tube. Dr. Galabin, when the same object was desired, adopted the plan of cutting a piece out of the tube between two ligatures, then pulling out the stump on each side as far as possible, and cutting it off so that the open lumen was left at the bottom of an inverted cone of cellular tissue. None of the patients so treated had become pregnant again, and he was inclined to regard this as a reliable method.

\section{ERYSIPELAS AND PUERPERAL FEVER.}

Is his Presidential Address before the Hunterian Society Dr. Galabin gave the results of a careful inquiry which he has made in regard to the annual variation of puerperal fever compared with that of certain allied diseases, namely, erysipelas, septicæmia and pyæmia, scarlet fever, and rheumatism. So far as can be seen by plotting out in graphic form the mortality from these several diseases, there appears to be but little relation between the annual prevalence of puerperal fever and that of either septicæmia, scarlet fever, or rheumatism, which is interesting when one remembers for how long a time the contagion of scarlet fever was held to be an im- 
portant cause of puerperal septicæmia. Not only is there but little similarity between the curves formed by plotting out the annual mortality of scarlet and puerperal fever for 20 years, but the curves formed by the mortality from these diseases, taken week by week, are absolutely different, scarlet fever rising to a maximum at the end of October and falling considerably before puerperal fever rises to its main maximum in early January. By far the most striking result of Dr. Galabin's inquiry is the accurate and precise manner in which the curves for erysipelas and for puerperal fever are shown to correspond, whether they are taken by the year or by the week, or are com- piled from the London statistics or from those of the country at large. In the annual curves almost every deviation in the one is represented in the other. A still more remarkable proof of the close relation of erysipelas and puerperal fever is to be found on comparing the curves of weekly variation. "Every curve and turn and every date when the curves cross the mean correspond. . . . This resemblance of the curves confirms the evidence of bacteriology as to the close connection between puerperal fever and erysipelas." The conclusion to be drawn is that the contagion of erysipelas is more dangerous to the puerperal woman than that of any other septic disease other than puerperal fever itself.

\section{Progress in Neurology.}

Tabes.-In a most useful paper Bramwell ${ }^{1}$ praises Frenkel's treatment of the ataxy of tabes and gives precise instructions as to how it is carried out. Frenkel pointed out in 1889 that in cases of tabes by the accurate repetition of a movement a notable diminution of the ataxia in association with that movement is produced, and this observation has been abundantly confirmed. The method is indeed one of re-education. The amount and rapidity of the improvement is largely influenced by the personal equation of the patient; the best results are obtained in persons who take an intelligent interest in the exercises and who are gifted with mental concentration and perseverance. The results are often striking; thus, a man who had been unable to walk or stand for three years was, after ten weeks' treatment, able to walk alone with a stick. A notable change in the patient's mental disposition often occurs ; from being depressed and miserable he becomes again hopeful and cheerful, owing to the improvement in the ataxy. The patient should be told that it is not the muscular force which he uses in performing the exercises which is of importance, but the care and precision with which he makes the individual movements. The exercises for the lower limbs are as follows : The patient lies on his back in bed and slowly raises his extended leg until he touches with his great toe the finger of the attendant held immediately above his foot, at a distance of 18 inches to 2 feet from the bed. Still lying on his back, he flexes the leg on the thigh to its full extent, and then the thigh on the abdomen, the whole limb is gradually extended until he touches with his great toe the finger of the attendant, which is held in the same position as in the previous exercise. Standing exercises are carried out by the patient being supported on either side, and then encouraged to practise his balancing power, gradually putting more and more weight upon his legs; he is also to practise standing with his feet close together, lifting one foot off the ground and placing it down again accurately. Walking exercises are performed by painting a black stripe 12 inches broad across the floor of a room, and along this the patient walks with support, taking care to keep his feet within its margins. Having mastered this exercise, he practises walking along a similar stripe on which, at distances of 1 foot, cross lines are painted, taking care to put his toe down exactly at the cross line. When these exercises are performed accurately, the strip may be made narrower -6 inches in breadth. Again, the patient must practise sitting down and getting up from his chair, walking up and down stairs, and so on. A quarter of an hour, two or three times a day, may be given to these exercises, and after each exercise the patient should take a short rest. It should be stopped on the earliest appearance of fatigue, or if there are any signs of the patient's attention beginning to wander.

Treatment of Hemiplegia.-Guthrie ${ }^{2}$ says that cases of hemiplegia are often not treated from the opinion that absolutely nothing can be done for them. Early and long-continued treatment, however, can do much-both hasten the recovery of mild cases and retard the absolute helplessness of severe ones. Thus, articular adhesions frequently cause limitation of movement. They may occur in the elbow, wrist, hip, knee, and ankle joints, but are most common, and are formed earliest, in the shoulder, rendering movements of the joint painful, if not impossible; and a limb which might have recovered is often useless owing to their presence. These adhesions can be prevented by passive movements of each joint from the first. Early rigidity, which so often limits movement, can be partially counteracted by stimulation of the opponents of the spastic muscles by massage. Thus, in the arm, the adductors of the shoulder, flexors of the elbow, pronators of the forearm, flexors of the thumb and fingers, adductor and opponens muscles of the thumb, are usually contracted, and should be left alone, whilst their opponents are regularly massaged and kept in good condition. Electricity, whether faradaism or galvanism, is a useful adjunct to massage, but cannot take its place. Re-education of movements is very necessary. A patient may not move his limbs, not so much because they are paralysed, but because he has forgotten how to do so. He must be taught, by executing the movement passively so that he may see how it is done, and getting him to try himself. Recovery naturally takes place first in the least specialised movements. The patient must therefore be taught to sit up before he can stand, stand before he can walk, move his shoulder before his elbow, and so on. Want of precision in movement often follows recovery, the regained movements are ataxic. Exercises on the Frenkel system, as employed in locomotor ataxy, are useful in such cases. This treatment of hemiplegia can be adopted quite irrespective of its cause, and need not interfere with other measures taken for the relief of the disease which gave rise to the hemiplegia.

1 Edin. Med. Jour., Sept.1901. 2 Lancet, Oct. 19, 1901. 